\title{
Gunther Tichy
}

\section{Erfordert die Informationsgesellschaft flexiblere Arbeitsmärkte?}

Wissenschaft, Politik und Wirtschaft stimmen weitgehend darin überein, dass die Zukunft in der New Economy liege, und dass diese erheblich flexiblere Arbeitsmärkte erfordere. Der deutsche Sachverständigenrat (2000, S. 218) fasst das unter dem Titel: „Anpassungen und Chancen in der Neuen Ökonomie“ zusammen: „Die Vorstellung von Arbeit als räumlich und zeitlich festgelegte, kontinuierlich abzuleistende Erwerbstätigkeit verliert an Bedeutung. ... [Es] werden sich zunehmend Beschäftigungsformen etablieren, die jenseits des traditionellen Arbeitsvertrags stehen." Manche Unternehmerkreise vermuten, dass in Zukunft nur noch $20 \%$ als Kernbelegschaft in feste Arbeitsverhältnisse eingebunden sein würden (Handy et al 1997; Föhrenbergkreis 1997). Was bedingt diesen radikalen Bruch mit der Vergangenheit, in der gerade langjährige Betriebszugehörigkeit honoriert wurde? Im folgenden wird zu zeigen versucht, dass zunehmende Komplexität die wichtigste Ursache dieses Gesinnungswandels ist, Flexibilisierung die Komplexität jedoch weiter vergrößert anstatt einen Beitrag zu ihrer Überwindung zu leisten. Was aber sind adäquate Strategien?

\section{Die unzureichende Begründung des Bedarfs an mehr Flexibilität}

Die meisten Begründungen des Bedarfs an mehr Flexibilität halten einer genaueren Überprüfung nicht stand. Dass die Zeit , raschlebiger ' geworden ist und man auf unvorhergesehene Ereignisse rascher reagieren müsse, lässt sich schwer messen, und die Einschätzung dürfte stark von subjektiven Elementen geprägt sein. Der technische Fortschritt (totale Faktorproduktivität) als messbarer Indikator des ,Tempos' hat sich in Europa in den neunziger Jahren deutlich verlangsamt und in den USA bloß die Verlangsamung in den siebziger und achtziger Jahren kompensiert (Scarpetta et al 2000). Bei der behaupteten Beschleunigung des Produktzyklus handelt es sich in den meisten Fällen bloß um marginale Marketing-induzierte Änderungen der Produkte (Leo 1999). Der Übergang zur New Economy als angeblich völlig neuem Paradigma hebt die ,basic economic principles' nicht auf; ${ }^{1}$ die Sekundärfolgen der Digitalisierung auf die anderen Branchen, insbesondere deren Organisation und Beschäftigung, werden allerdings zur zunehmenden Komplexität beitragen, gegen die Flexibilisierung - wie zu zeigen versucht wird - jedoch keine eine adäquate Strategie ist.

Kann - auf operationaler Ebene - weitgehendes Outsourcing und völlige Flexibilisierung der Arbeitsverhältnisse als Übergang von hierarchischen Mechanismen (Organisation) auf Marktbeziehungen die Allokation verbessern? Nach der Theorie des Unternehmens ${ }^{2}$ ist hierarchische Organisation marktmäßiger dann überlegen, wenn es um spezialisierte Produktionsmittel und seltene bzw. komplexe Transaktionen geht, ${ }^{3}$ der Erfolg der Tätigkeit nicht leicht zu bewerten ist, die Transaktionen eng miteinander verflochten sind, oder eine Weitergabe von Information unerwünscht ist ${ }^{4}-$ Voraussetzungen deren Bedeutung eher zu- als abnimmt. Dem entspricht die Beobachtung, dass Unternehmensgröße und Komplexität in der Vergangenheit markant zunahmen, der Anteil der Markttransaktionen mit steigender Komplexität also abnahm. Auch steht die Marktideologie der strikten Flexibilisierung in markantem Gegensatz zu dem tatsächlichen Verhalten der Unternehmen am Markt: Diese tendieren - vor allem durch Fusionen - immer größer zu werden um weltweit zu

1 "Ignore basic economic principles at your own risk. Technology changes. Economic laws do not." (Shapiro and Varian 1998). Manche der neueren Untersuchungen schätzen die Folgen der Informationstechnologie deutlich weniger revolutionär ein, als die Erfindungen in der zweiten Hälfte des 19. Jahrhunderts (Gordon 2000), vor allem wenn man auch die organisatorischen Innovationen berücksichtigt (z.B. Fordismus, Divisionalisierung, Versandhandel).

2 Siehe etwa Milgrom and Roberts 1992.

3 Ein Zulieferer wird nicht bereit sein, teure, bloß für einen Kunden nutzbare Investitionen zu tätigen, da er damit rechnen muss, nach der Investition unter erheblichen Preisdruck seines Kunden zu geraten ('ex-post opportunism').

4 Etwa bei der Entwicklung neuer Produkte. Effiziente Märkte setzen eine große Zahl von Käufern und Verkäufern für dasselbe - standardisierte - Produkt voraus; je geringer deren Zahl bzw. Nachfrage, je spezifischer das Produkt, desto dünner der Markt, desto höher die Suchkosten für die Beteiligten und desto ineffizienter der Markt. 
den jeweils größten Drei zu gehören. Zunehmend hierarchische Steuerung verdrängt Markttransaktionen und schafft eine eng oligopolistische Marktstruktur.

Der vermutete unmittelbare Zusammenhang zwischen Flexibilisierung und Effizienz vernachlässigt die langfristigen Bestimmungsgründe der Effizienz: Betriebsklima, Akkumulation unternehmensspezifischer Fähigkeiten, Bildung entsprechender Netzwerke oder Redundanz. "Corporate myopia” (Swann 1998, S. 109) übersieht, dass kurzfristige Effizienzsteigerung langfristige vielfach ausschließt.

\section{Die tieferliegenden U rsachen des Suchens nach neuen Konzepten}

Wichtigste Ursache für die Suche nach neuen Konzepten ist die zunehmende Komplexität, mit der Wirtschaft und Wirtschaftspolitik konfrontiert sind; sie hat mehrere Determinanten:

An erster Stelle die zunehmende Spezialisierung der Information, die laufend neue Fachgebiete entstehen lässt und Kooperationen und Netzwerke erzwingt. ${ }^{5}$ Tätigkeiten werden ausgelagert, weil in Spezialfirmen konzentrierte Spezialisten ihr Spezialwissen vertiefen und Größenvorteile nutzen, somit effizienter eingesetzt werden können. Allerdings erhöht radikale Arbeitsteilung - auch im geistigen Bereich - die Komplexität durch Zwang zur Interdisziplinparität und Kooperation.

Die zunehmende Spezialisierung resultiert primär aus dem kumulativen Charakter des Wissens. Obwohl neue Information alte vielfach obsolet oder trivial macht, wächst die Informationsmenge an, die nicht bloß gelernt und in Evidenz gehalten sondern auch reflektiert werden muss. Je schmaler jedoch das Gebiet, auf dem der Spezialist über reflektiertes Wissen verfügt, desto mehr bedarf er der Kooperation mit anderen Spezialisten, mit allen Komplexitätsproblemen der interdisziplinären Zusammenarbeit.

Die Spezialisierung des Wissens ergibt sich aus der zunehmenden Differenzierung der Aufgaben: Kein Unternehmen kommt mehr mit einfacher Mechanik aus; elektronische Steuerung, Mess- und Regeltechnik, Simulation, etc. erfordern aber nicht bloß zusätzliche Spezialkenntnisse, sondern vielfach auch deren Kombination; ${ }^{6}$ die Technik ist unsichtbar, komplexer und Spezialisten-abhängiger, der Spezialist Technik-abhängiger geworden.

Über die technische Komplexität hinaus hat auch die organisatorisch-führungstechnische Komplexität zugenommen. Kostendruck zwingt die Unternehmen immer komplexere organisations- oder informationstechnische Instrumente zur Sicherung der Kapazitätsauslastung (Flexibilität), sowie der Rationalisierung von Zulieferung, Logistik und Vertrieb einzusetzen, die nur von Spezialisten entwickelt und betreut werden können. ${ }^{7}$ Auch die rasant zunehmende Größe und Internationalisierung der Firmen vergrößert die Komplexität effizienter Führung; sie erfordert zunehmend komplexere Instrumente.

Ähnlich wie die Aufgaben der Firmen haben sich die Bedürfnisse der Kunden zunehmend differenziert. ${ }^{8}$ Mit zunehmendem Wohlstand gewannen Erfahrungsgüter, deren Qualität erst durch längeren Gebrauch erkannt werden kann an Bedeutung; die Marktreaktionen treten dadurch verzögert auf und sie wurden erratischer.

Die Mikroelektronik hat viele der erwähnten Entwicklungen ermöglicht, indem sie der Großindustrie den Weg in die diversifizierte, qualitativ hochwertige Kleinserienproduktion öffnete. Einerseits wurde der Wettbewerb dadurch schärfer, andererseits mussten alle Unternehmen zusätzliche Kompetenzen in Elektronik

5 Die Zulieferung zahlreicher Komponenten eines Kraftfahrzeugs durch Spezialisten resultiert weniger aus Rationalisierung als aus Spezialisierung: das Spezialwissen für Entwicklung und Erzeugung ist in der Automobilfabrik nicht vorhanden, und zu vertretbaren Kosten nicht erwerbbar. Ähnlich liefern EDV-Spezialisten, Kostenrechnungs-Spezialisten, Steuer-Spezialisten, Organisations-Spezialisten dem administrativen und organisatorischen Bereich zu.

6 Komplexität bedeutet, dass ein einfacher robuster selbst in Entwicklungsländern leicht und rasch entwickelt werden kann; die Entwicklung eines zugleich sparsamen, abgasarmen, vibrationsfreien, leisen und dauerhaften Automotors bedeutet hingegen eine Optimierungsaufgabe, einen Spezialisierungsgrad und einen Finanzbedarf, der allein von einigen wenigen hochspezialisierten Entwicklungsfirmen geleistet werden kann.

7 So wirft etwa die Anpassung an die durch Informationstechnik ermöglichte genaue Kenntnis der spezifischen Wünsche der Kunden komplexe Entscheidungsprobleme in Bezug auf Produktionssteuerung und Logistik auf.

8 Ford konnte sein Modell T allein in Schwarz anzubieten, Volkswagen mit einem einzigen, im Zeitverlauf bloß marginal veränderten Modell jahrzehntelang Marktführer bleiben. Heute müssen unzählige Varianten desselben Massenmodells (im Kfz-Bereich ,Plattform-Strategie') angeboten werden, die höchst spezialisierte Anforderungen an die (flexible) Massenproduktion und, mehr noch, an die Logistik der Firmen stellen 
wie in Unternehmensorganisation und der Logistik erwerben. Der Komplexitätsgrad stieg dadurch beträchtlich an.

Die Informationsintensität der Wirtschaftsprozesse ist weiters durch die Verwissenschaftlichung der Innovation gestiegen. Statt des linearen Innovationsprozesses - von der Invention aus weitgehend autonomer Forschung oder Zufall über Innovation zur Produktion - erzwingt der verstärkte Wettbewerb systematische laufende Innovation: Wissenschafter wie Firmen forschen unmittelbar anwendungsorientiert; vielfach lässt sich Grundlagenforschung von Umsetzung nicht mehr unterscheiden. ${ }^{9}$ Höchste Spezialisierung der Wissenschafter, ihrer Auftraggeber und der Financiers, ist in diesem Prozess zentral; die Fähigkeit kontinuierlich Sequenzen miteinander verbundener, synergistischer Innovationen hervorzubringen, ist eine schwierige Unternehmeraufgabe von höchstem Komplexitätsgrad.

Schließlich sind die Rückkopplungseffekte zu beachten: Wenn ein Wirtschaftssubjekt in einer interdependenten Welt sein Verhalten ändern muss, ändert das die Handlungsvoraussetzungen für die anderen und zwingt diese zu Reaktionen. In einer komplexen Welt ist jedoch nicht bloß eine einzige, für die Anderen abschätzbare Reaktion möglich, sondern unzählige. Daraus entsteht Unsicherheit der Betroffenen, die sie zu einer Anpassung ihrer Strategie in einer Form zwingt, die viele Reaktionsmöglichkeiten offen hält, woraus weitere Komplexität für die dadurch Betroffenen entsteht: "Increasing complexity feeds on itself, generating more complexity in the process." (Trisoglio 1995, S. 8).

Größere Komplexität, das Resultat dieser Entwicklungen bedeutet, dass sich Phänomene nicht auf einfache Ursachen zurückführen lassen, Nicht-Linearitäten an Bedeutung gewinnen, und Daten der Vergangenheit nur begrenzt Schlussfolgerungen für Gegenwart und Zukunft erlauben. Optimierung im Rahmen einer stabilen prognostizierbaren Entwicklung ist jedoch die Strategie, die die Firmen in der weitgehend strukturkonstanten Vergangenheit verinnerlicht haben; sie impliziert i.d.R. verminderte Anpassungsfähigkeit an neue Entwicklungen. Die Lösung des Komplexitätsproblems muss daher in einer weiter gehenden Reform der Strategie gesucht werden: Komplexe Systeme „müssen mit internen Unwahrscheinlichkeiten und Unzulänglichkeiten zurechtkommen. ... Komplexe Systeme sind mithin zur Selbstanpassung gezwungen, und zwar im Doppelsinne einer eigenen Anpassung an die eigene Komplexität." (Luhmann 1988, S. 56, meine Hervorhebung G.T.).

Neu ist somit die gestiegene Komplexität, der Manager wie Politiker gegenüberstehen und, als Reaktion darauf, die generelle Tendenz zu kurzfristiger Perspektive. Die neue Komplexität hat nichts mit dem Tempo des Wandels zu tun; auch rascher Wandel ist beherrschbar, wenn die Richtung der Änderung vorhersehbar ist. Komplexität bedeutet jedoch, dass die Richtung der Änderungen nicht ohne weiteres vorhersehbar ist, und dass mögliche Alternativen nicht leicht auf eine gemeinsame Basis gebracht werden können; es gilt nicht bloß Risken abzuwägen, es geht um Entscheidungen unter Unsicherheit. ${ }^{10}$

\section{Die mangelnde Berücksichtigung von Komplexität in den traditionellen Unternehmensstrategien}

Da zunehmende Komplexität längerfristige Planung traditioneller Art verhindert, macht sie klassische Managementinstrumente, wie Management by Objetives oder Planning, Programming, Budgeting obsolet, die von einer linearen, mechanistischen Entwicklung ausgehen. Für diese ist Management ein Prozess der Planung, Organisation, Koordinierung, Anordnung und Kontrolle; ihre Ziele Effizienz und Optimierung, stehen in einer komplexen, vernetzten Welt jedoch zweitrangig hinter der Entwicklung einer Strategie, die technologische und Markttrends nutzt. Auch diesbezüglich versagen die üblichen Instrumente:

Die Strategie der strikten Orientierung an den Kundenwünschen reduziert zwar Komplexität, da sie, passiv reagierend, das Problem abschiebt: Kunden wüssten selbst am besten, wie sich ihre Märke entwickeln. Ohne Kenntnis der neuen Möglichkeiten können Kunden aber gar nicht wissen was sie wollen, und die Entwick-

9 Start-up-Firmen werden heute vielfach gegründet bevor die Idee marktreif ist - hier gibt es die Beschleunigung, die beim technischen Fortschritt nicht zu erkennen ist; neue Produkte entstehen allerdings zumeist auch nicht rascher, wie das Beispiel der Gentechnik oder die jüngste Krise der dot.com-Firmen illustrieren.

10 Die aus dem Übergang zu netzwerkartigen Strukturen resultierende , winner takes it all'-Gefahr verschärft das Entscheidungsproblem weiter. Die Reaktion, sich unter diesen Voraussetzungen alle Möglichkeiten offen zu halten, möglichst große operative Flexibilität zu schaffen, ist nur zu verständlich. 
lungszusammenarbeit mit ihnen unterliegt der Gefahr des Nutzer-Paradoxons (Tichy 2001), der Vernachlässigung radikaler Innovationen zugunsten inkrementaler: Die Kunden sind primär an Verbesserungen ihrer Produkte interessiert, radikale Neuerungen kommen i.d.R. von neuen Firmen.

Konzepte wie Wertanalyse oder Return on Investment (ROI) liefern bei zunehmender Komplexität sogar irreführende Ergebnisse. Sie betrachten das Unternehmen nicht als komplexes System und vernachlässigen daher gerade die zentralen Ursachen der Komplexität: Verbundeffekte, Synergien, oder intertemporale Interdependenz. ${ }^{11}$

Auch die externe Kontrolle der Unternehmensstrategien durch den Kapitalmarkt (Shareholder Value, Market for Corporate Control) kann das Komplexitätsproblem nicht lösen. In der Theorie prämiert sie diejenigen Firmen, die das Problem gelöst haben, in der Praxis führt sie systemimmanent zu kurzfristiger Disposition zwecks Ertragsmaximierung; ${ }^{12}$ bei komplexen Entscheidungen kann das jedoch das Überleben gefährden.

Mangels anderer Instrumente erscheint Flexibilität aller Unternehmensbereiche somit unvermeidlich, um in einer nicht prognostizierbaren Welt auf Unvorhergesehenes reagieren zu können. Allerdings besteht die Gefahr, dass Strukturen, die rasche Reaktionen ermöglichen (Flexibilisierung), die Anpassung an grundlegende Änderungen erschweren. Demgemäss erscheint es zweckmäßig, in der Tradition von Klein (1984) zwei Arten von Flexibilität zu unterscheiden: Flexibilität I als Fähigkeit auf Änderungen (Risken) rasch zu reagieren, und Flexibilität II als Fähigkeit neu auftretende Chancen nutzen zu können; bei ersterer geht es primär um Kosten, wie Abbildung 1 in Anlehnung an Stigler (1939) illustriert: Kurve A spiegelt eine Faktorkombination (Technologie), mit der eine bestimmte, eng begrenzte Outputmenge oder -variation mit minimalen Kosten produziert werden kann; wird diese über- oder unterschritten steigen die Kosten rasch an, die Flexibilität ist also gering. Kurve B spiegelt (bei gegebenen Preisen) eine Situation sehr viel größerer Flexibilität, zeigt allerdings auch die Kosten, mit der diese Flexibilität erkauft werden muss: Zwischen X und Y produziert sie erheblich teurer. Was üblicherweise unter Flexibilisierung verstanden wird, entspricht Flexibilität I, dem Übergang von Kurve A zu Kurve B. Flexibilität II hingegen, als "possibility to move to various second period positions" (Jones and Ostroy 1984), als Fähigkeit neu auftretende Chancen überhaupt nutzen zu können, hat weniger mit den Kosten und der jeweiligen Kurvenform zu tun, als mit der Fähigkeit Chancen zu erkennen, sie umzusetzen, und durch die Einzigartigkeit des Angebots einen Preissetzungsspielraum zu schaffen. Flexibilität II eröffnet auf längere Sicht größere Chancen.

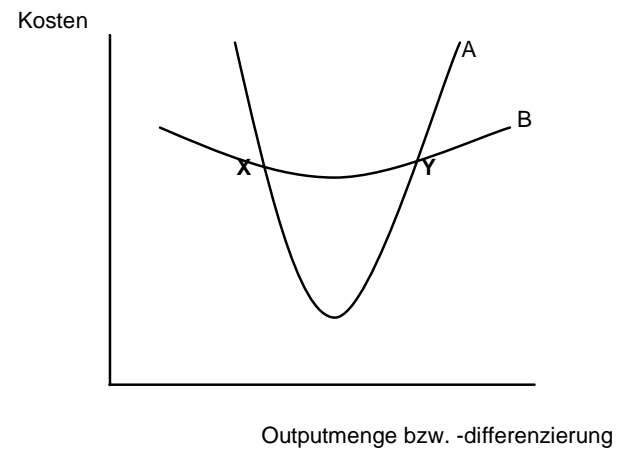

Abbildung 1: Outputflexibilität

11 Siehe dazu etwa Schmidt und Maßmann 1999.

12 Der Ansatz setzt voraus, dass auf dem Aktienmarkt hochinformierte Personen agieren, die ihr eigenes Vermögen anlegen; tatsächlich jedoch fühlen sich die meisten Anleger zu wenig informiert und delegieren die Anlageentscheidung. Fast alle Akteure am Aktienmarkt legen daher fremdes Geld an, und sind in ein mehrgliedriges hierarchisches System eingebunden. Die Angestellten, die die Anlageentscheidungen treffen, können Komplexität und langfristige Aspekte nicht berücksichtigen; sie müssen, wenn nicht vierteljährlich, so doch innerhalb eines Jahres gegenüber ihren Vorgesetzten - und die Bank gegenüber ihren Kunden - Erfolge in Form von laufenden Erträgen nachweisen. 


\section{Ansatzpunkte für eine Strategie des Umgangs mit Komplexität}

Eine inzwischen breit angewandte, Teilerfolge versprechende Strategie ist die Konzentration auf die Kernkompetenzen, die 'specific assets'. ${ }^{13}$ Sie reduziert Komplexität durch Spezialisierung und erleichtert zugleich die Vertiefung der Kernkompetenzen. Allerdings impliziert Spezialisierung auch die Gefahr eines 'lock in' in nicht mehr marktgängige 'specific assets' und das ,potentielle ,Aushöhlen' des Unternehmens; denn durch pauschales Outsourcing werden sämtliche mit dieser Tätigkeit zusammenhängenden internen Kompetenzen und Fähigkeiten und alles Gelernte abgetreten.“ (Mogensen und Thumm 2000, S. 39). Offen bleibt auch, wie die Firma Kernkompetenzen entwickelt, die anpassungsfähig und zukunftssicher sind. Dieser Ansatz bedarf daher zumindest ergänzender Strategieelemente.

Ausgangspunkt für einen strategischen Umgang mit Komplexität muss eine systemtheoretische Perspektive sein, "a shift of mind - seeing interrelationships rather than linear cause-effect chains, and seeing processes of change rather than snapshots" (Senge 1990, S. 73), sowie der Ersatz der Optimierungsstrategie durch eine Überlebensstrategie. Überleben in einer komplexen Welt erfordert jedoch Flexibilität II, als Fähigkeit neu auftretende Chancen überhaupt nutzen zu können; Elastizität, Anpassungsfähigkeit, aktives Streben nach Veränderung und organisatorisches Lernen sind dafür zentrale Voraussetzungen.

\subsection{Institutionelle Voraussetzungen: Elastizität und Anpassungsfähigkeit}

Elastizität ist sowohl für die Strategie als auch für die Struktur der Firma erforderlich. Die Strategie muss elastisch sein, damit die Firma auf Schocks rasch und richtig reagieren kann, die Struktur, um die Schwäche oder den Ausfall eines Elements entsprechend kompensieren zu können. Eine Möglichkeit Elastizität zu gewinnen ist Redundanz (Grabherr o.J.), was zwar in der Informations- ${ }^{14}$ wie in der Unternehmenstheorie ${ }^{15}$ gleichbedeutend mit Ineffizienz ist; in komplexen Systemen jedoch verringert Redundanz der Information, der Strukturen, der Funktionen oder der Beziehungen die Fehleranfälligkeit und eröffnet Alternativen, wenn unvorhergesehene Ereignisse den zunächst gewählten Weg unattraktiv erscheinen lassen. Vor allem in Systemen, die aus wenig verlässlichen Teilsystemen bestehen, sichert sie ein hohes Maß an Verlässlichkeit; die Wahrscheinlichkeit eines Fehlers fällt mit zunehmender Redundanz exponentiell (Landau 1969, S. 350).

Parallel- oder Reservesysteme sind eine spezifische Form der Redundanz, die primär der Funktionssicherheit dienen; sie können aber auch zur Überlebenssicherung des Systems eingesetzt werden, etwa in Form der (vielfach verpönten ${ }^{16}$ ) Parallel- oder Doppelentwicklungen: Werden zwei oder mehrere Teams auf dasselbe Problem angesetzt, wirkt nicht bloß die Konkurrenz stimulierend; auch die Wahrscheinlichkeit unterschiedlicher Ergebnisse steigt, und damit die Wahlmöglichkeiten des Auftraggebers wie die Elastizität. Das Risiko, dass der Pfad der lokalen Maximierung in einer Sackgasse endet, wird reduziert (Simon 1983, S. 66ff). Weitere Möglichkeiten einer Erhöhung der Elastizität liegen in einer laufender Fehlerkontrolle und im Monitoring, in der Dezentralisierung von Entscheidungen und Prozessen, wie in einer Job rotation, die die Mitarbeiter flexibel verwendbar macht. Gut funktionierende, langfristig konzipierte Netzwerke mit Zulieferern, Vertriebspartnern und Servicefirmen können weitere Maßnahmen zur Erhöhung der Elastizität sein.

Elastizität als eher statisches Konzept mag noch gewisse Ähnlichkeit mit den Flexibilisierungsstrategien gemäß Flexibilität I haben, wenn auch Redundanz oder Parallelsysteme infolge ihrer Kosten bei Rationalisierungsexperten keineswegs hoch im Kurs stehen; Anpassungsfähigkeit, das zweite wichtige Strategieelement in einer komplexen Welt, hingegen steht in diametralem Gegensatz zu den üblichen betriebswirtschaftlichen Strategien. Die zentralen Ideen hinter dem Begriff Anpassungsfähigkeit sind Diversifizierung und Umgang mit Vielfalt bzw. Verschiedenheit, Konzepte, die Voraussetzungen dafür schaffen, bei Änderungen des Marktes unter mehreren Alternativen wählen zu können. Das wiederum ist nur möglich, wenn Redundanz toleriert und Parallelentwicklungen nicht bloß zugelassen sondern forciert werden. Flache Hierarchien, mo-

13 Zum Begriff der asset specifity siehe etwa Milgrom and Roberts 1992, S. 307.

14 "Zero redundancy constituted the measure of optimal efficiency." (Landau 1969, S. 348)

15 „Alle strukturierenden Regelungen sind danach zu beurteilen, inwieweit sie mit einem Minimum an eingesetzten Gütern (Sachgüter, Arbeits- und Dienstleistungen etc.) der Aufgabenerfüllung dienen“ (Kosiol 1962, S. 25)

16 Siehe etwa die Bemühungen der EU durch Koordinierung der Forschung zu vermeiden, daß in zwei Staaten an demselben Problem gearbeitet wird - einer der Versuche Redundanz zugunsten kurzfristiger Effizienz zu verringern. 
dularer Aufbau der Firma, dezentrale Entscheidungen, lokale Kontrolle und kritische Auswertung der Erfahrungen ('after action reviews') sowie Förderung der horizontalen Zusammenarbeit sind wichtige organisatorische Voraussetzungen für eine entsprechende Anpassungsfähigkeit der Organisation.

Noch eine Stufe weiter als das eher passive Konzept der Anpassungsfähigkeit geht die Strategie der bewussten Suche nach Veränderung. Sie hat zwei Komponenten: Eine bewusste Distanzierung von der jeweils gängigen Praxis: “... one reason adaptation may preclude adaptability is that people remember only those practices that are currently useful. Memory may preclude innovation." (Weick 1979). Zweitens muss Veränderung aktiv gesucht werden, da bestehende Firmen zumeist in ihrer Normaltätigkeit gefangen sind: Markante Neuerungen erwerben sie zumeist durch Akquisition dynamischer junger Firmen, einschneidende Reorganisationsmaßnahmen oder neue Verfahren setzen zumeist negative Schocks voraus (Cooper and Haltiwanger 1993; Aguirregabiria and Alonso-Borrego 2001). Um endogen änderungsfähig zu sein, müssen Firmen unkonventionelle Nebenaktivitäten ('active fringe') zulassen - neue Ideen kommen zumeist von außen, durch Kontakte mit Personen aus anderen Branchen, mit anderen Auffassungen und Konzepten (Noteboom 1999). Die Firmen müssen Fähigkeit wie Bereitschaft entwickeln, unkonventionelle ,Außenseiter in der Firma' zu fördern, sich mit deren Ideen auseinanderzusetzen, passende aufzugreifen und umzusetzen. Einer Strategie der strikten Konzentration auf Kernaktivitäten widerspricht das.

\subsection{Corporate Vision und organisatorisches Lernen als Instrumente}

Der Rahmen der institutionellen Voraussetzungen zur Erreichung von Flexibilität II muss durch konkretes Handeln der Firma aufgefüllt werden, um Beziehungsnetzwerke aufzubauen und die Prozesse erkennen zu können, die die künftige Entwicklung bestimmen. Ausgangspunkt dafür muss eine Corporate vision sein, eine Vorstellung über Form, Position und Aufgaben der Firma in der Zukunft. ${ }^{17}$ Die Maßnahmen zur Erhöhung der Flexibilität II sollten auf der capability-Theorie basieren; sie sieht die Firma als ein Bündel spezifischer Fähigkeiten (Metcalfe and de Liso 1998, S. 19), die die die Firma als 'strategic assets' gezielt aufbauen muss, um mit Unsicherheit zurecht zu kommen. Die capabilities mögen zum geringen Teil in Patenten und Firmenhandbüchern kodifiziert sein, überwiegend bestehen sie aus dem Wissen der Mitarbeiter, und vielfach handelt es sich um 'tacit knowledge', Wissen das nicht ohne weiteres kodifiziert und weitergegeben werden kann. Insofern bilden die Mitarbeiter mit ihrem individuellen Wissen im Rahmen der Organisation der Firma und mit Hilfe komplementärer, vielfach firmenspezifischer Produktionsfaktoren die capability der Firma als "competence pool" (McKelvey 1982). Sie zu vertiefen, muss die Strategie des 'planned change' der siebziger Jahre einer Strategie des 'managed learning' und der Förderung der Kreativität weichen. "It's just not possible any longer to 'figure it out' from the top" und daher unverzichtbar, "to discover how to tap people's commitment and capacity to learn at all levels." (Senge 1990, S. 4).

Die gezielte Bildung firmenspezifischen Humankapitals muss von der Firma bewusst gestaltet werden, da für die Mitarbeiter selbst allgemeines, firmenunspezifisches Humankapital wertvoller ist: es macht sie krisenresistenter und erhöht ihre Mobilität. ${ }^{18}$ Firmenspezifisches Wissen, das zugleich eine enge Firmenbindung schafft, kann vor allem durch firmeninterne Aus- und Weiterbildung vermittelt werden. "The ability of the firm to absorb and to act upon knowledge depends in part on staff continuity. Practical steps to achieve this include on-the-job promotion and share options, or, more subtly, technology can be deployed strategically to 'lock-in' staff. Corporate vision can add as a focusing device." (Swann, 1998, S. 118). Hire and fire-Strategien wirken dem Erwerb firmenspezifischen Humankapitals entgegen, ${ }^{19}$ ebenso Verunsiche-

17 Das widerspricht nicht der grundsätzlichen Unprognostizierbarkeit komplexer Systeme; allerdings kann es sich nicht um determinististische Prognosen ('forecast') handeln, sondern um 'foresight', um die probabilistische Einschätzung künftiger Entwicklungen. Eine solche ist als Basis des organisatorischen Lernens der Firma unverzichtbar. Entscheidend sind weniger die Ergebnisse, als das damit verbundene organisatorische Lernen, die systematische Beschäftigung mit unterschiedlichen längerfristigen Entwicklungspotentialen; dadurch bekommen die Mitarbeiter ein Gefühl für die komplexe Entwicklung, in die die Firma eingebettet ist, für deren Dynamik und für mögliche Alternativen.

18 Die Flexibilisierungsstrategie (I) der bloß temporären Beschäftigung erzieht egoistische Mitarbeiter; nur Mitarbeiter, die sich mit der Firma identifizieren, eine langfristige Firmenbindung anstreben und in der Firma Karriere machen wollen, werden bereit sein, firmenspezifisches Humankapital zu erwerben

19 Das gilt in besonderem Maße für Europa; in den USA hingegen erfolgen die Freisetzungen vielfach bloß temporär, d.h. daß die Freigesetzten bei Änderung der Lage bevorzugt wieder eingestellt werden. 
rung der Mitarbeiter durch häufigen Strategiewechsel der Firma als Folge wechselnder Manager oder Eigentümer. Bloß temporäre Verträge können besonders kontraproduktiv wirken, da die temporär Beschäftigten daran interessiert sein werden, gerade die strategischen Kompetenzen der Firma kennen zu lernen, deren Kenntnis ihre Beschäftigungschancen in Konkurrenzfirmen verbessert.

Flexibilisierung im Sinn von Flexibilität I schafft somit zwar den Spielraum für rasches Reagieren, der für rasche Anpassungen (auch des Personals) zuweilen auch erforderlich ist. Es besteht jedoch ein tradeoff zu Flexibiltät II, der Fähigkeit Existenzgefährdungen nachhaltig abzuwehren und neue auftretende Chancen entsprechend nutzen zu können. Letzteres erfordert firmenspezifische Kompetenz, spezifische Fähigkeiten, über die die Konkurrenten nicht verfügen, und die durch ad hoc-Zukauf von Experten, Expertise und Vorleistungen vielleicht erweitert, nicht jedoch geschaffen werden können.

Dieselben Argumente wie für die Bindung der Mitarbeiter zum Aufbau firmenspezifischer assets gelten für längerfristige Vertragsbindungen gegenüber Zulieferern oder eigenständigen Vertriebsunternehmen. Je kürzer diese Bindungen, je leichter kündbar, desto mehr müssen die Zulieferer auf breite Verwendbarkeit ihrer Investitionen und Entwicklungsaufwendungen zu achten, und desto weniger werden sie bereit sein für ihren (möglicherweise bloß temporären) Kunden spezifische Varianten zu entwickeln, womit dessen Chance sinkt, sich mit seiner Produktionspalette von den Konkurrenten markant abzuheben.

\section{$5 \quad$ Ausblick}

Der Capability-Ansatz zeigt, dass der Verzicht auf Flexibilisierung im Sinne eines Verzichts auf kurzfristige Reaktionsmöglichkeiten (Flexibilität I) zugunsten langfristiger Personalpolitik und Firmenbindung dem Aufbau firmenspezifischer Kompetenz (Flexibilität II) erheblich nützt; kurzfristige Verträge vermeiden zwar Bindungen, hindern aber die Entwicklung von Alternativen zu den jeweiligen Strategien. Noch problematischer als auf Firmenebene ist Flexibilisierung (I) auf volkswirtschaftlicher Ebene: Die Verringerung der firmenspezifischen Kompetenz erschwert den volkswirtschaftlichen Strukturwandel; der Verzicht auf langfristige Personalbindung reduziert nicht bloß die Ausbildung durch die Firmen, sondern auch die individuellen Anreize zur Aus- und Weiterbildung durch Verunsicherung und kurzfristige Orientierung; Fachkräftemangel ist die unvermeidliche - und bereits sichtbare - Folge. Dazu kommt, dass die rasch alternde Bevölkerung ohnedies risikoscheuer, weniger lernfähig und strukturkonservativer wird. Diejenigen Firmen, die sich schon jetzt auf die Änderung der Voraussetzungen einstellen und wieder Instrumente entwickeln, um eine Stammbelegschaft mit firmenspezifischem Wissen aufzubauen und längerfristig zu halten, werden einen nicht unerheblichen Konkurrenzvorsprung erlangen. Eine Wirtschaftspolitik, die diese Tendenzen vorwegnimmt, bewusst macht, und die entsprechenden Rahmenbedingungen schafft, kann zur Sicherung der Konkurrenzfähigkeit und Standortqualität der betreffenden Landes erheblich beitragen. 


\section{Literatur}

Aguirregabiria, V. and C. Alonso-Borrego (2001), Occupational structure, technological innovation, and reorganization of production, Labour Economics 8, 43-73.

Cooper, R. and J. Haltiwanger (1993), The aggregate implications of machine replacement: theory and evidence, American Economic Review 83, 360-82.

Föhrenbergkreis (1997), Jobshift. Überlegungen zur neuen Welt der Arbeit, IWIP Institut für Arbeit und Politik, Wien.

Gordon, R.J. (2000(, Does the ,New Economy“ measure up to the great inventions of the past? NBER-Working Paper No.W7833.

Grabherr, G. (о.J.), Zur Ökonomie des Überflusses: Die Bedeutung von Redundanz für die Regionalentwicklung, Manuskript, Wissenschaftszentrum Berlin.

Handy, Ch., et al (1997), Rethinking the future. Business, principles, competition, control, leadership, markets and the world, N. Brealey, London.

Jones, R.A. and J.M. Ostroy (1984), Flexibility and uncertainty, Review of Economic Studies 51, 13-32.

Klein, B.H. (1984), Prices, wages, and the business cycle: A dynamic theory, Pergamon, New York.

Kosiol, E. (1962), Organisation der Unternehmung, Gabler, Wiesbaden.

Landau, M. (1969), Redundancy, rationality, and the problem of duplication and overlap, Public Administration Review $29,346-58$

Leo, H. (1999) ,Österreichs Innovations- und Forschungsleistung im internationalen Vergleich', Monatsberichte des Österreichischen Instituts für Wirtschaftsforschung 72, 435-43.

Luhmann, N. ('1988), Soziale Systeme. Grundriß einer allgemeinen Theorie, Suhrkamp, Frankfurt.

McKelvey, W. (1982), Organizational Systematics, University of California Press, Berkeley.

Metcalfe, St. and N. de Liso (1998), Innovation, capabilities and knowledge: the epistemic connection, in R. Coombs et al, (eds), Technological change and organization, Elgar, Cheltenham/Northampton, MA., 8-27.

Milgrom, P. and J. Roberts (1992), Economics, organization \& management, Prentice Hall, Upper Saddle River, NJ.

Mogensen, J. und N. Thumm (2000), Outsourcing von Geschäftsdienstleistungen, IPTS Report 49, 36-44.

Noteboom, B. (1999), Innovation and inter-firm linkages: new implications for policy, Research Policy 28, 793-805.

Sachverständigenrat zur Begutachtung der gesamtwirtschaftlichen Entwicklung (2000), Chancen auf einen höheren Wachstumspfad. Jahresgutachten 2000/01, Metzler-Poeschel, Stuttgart.

Scarpetta, St. et al (2000), Economic growth in the OECD area: Recent trends at the aggregate and sectoral level, $O E C D$ Working Papers 248, OECD, Paris.

Schmidt, R.H. und J. Maßmann (1999), Drei Mißverständnisse zum Thema 'Shareholder Value', in Kumar, B. et al, Hg, Unternehmensethik und die Transformation des Wettbewerbs, Schäffer/Poeschel, Stuttgart, 125-151.

Senge, P.M. (1990), The fifth discipline: The art and practice of the learning organization, Doubleday, New York.

Shapiro, C. and H. Varian (1998), Information rules. A strategic guide to the network economy, Harvard Business School, Boston, http://www.inforules.com.

Simon, H., 1983, Reason in human affairs, Blackwell, Oxford.

Stigler, G. (1939), Production and distribution in the short run, Journal of Political Economy 47, 305-27.

Swann, P. (1998), Rapid technological change and shortening business horizons, in R. Coombs et al, eds, Technological change and organization, Elgar, Cheltenham/Northampton, MA., 108-23.

Tichy, G. (2001), Das Nutzer-Paradoxon und seine Bedeutung für die europäische Innovationsschwäche. Neue Ansatzpunkte für die Technologiepolitik? in W. Fuchs und O. Horvath, Hg, Wirtschaftsstandort Österreich. Von der Theorie zur Praxis, Bundesministerium für Wirtschaft und Arbeit, Wien, 207-30.

Trisoglio, A.,(1995,) Complexity: The challenges, presented at the workshop 'Risk, Policy and Complexity, mimeo, IIASA.

Weick, K.E. (1979), The social psychology of organizing, Addinson-Wesley, Reading, Mass. 